\title{
The Experiences of Patients with Deep Brain Stimulation in Parkinson's Disease: Challenges, Expectations, and Accomplishments
}

\author{
Özlem İbrahimoğlu' ${ }^{1}$, Sevinç Mersin², Eda Akyol $^{3}$ \\ ${ }^{1}$ Nursing Department, Faculty of Health Sciences, İstanbul Medeniyet University, İstanbul, Turkey, ${ }^{2}$ Nursing Department, \\ Faculty of Health Sciences, Bilecik Seyh Edebali University, Bilecik, Turkey, ${ }^{3}$ Nursing Department, Faculty of Health Sciences, \\ İstanbul Medeniyet University, İstanbul, Turkey.
}

Correspondence: oogutlu@gmail.com; Tel.: + 90216280 2754; Fax.: + 902282141382

Received: 19 August 2019; Accepted: 12 March 2020

\begin{abstract}
Objectives. Deep brain stimulation (DBS) is a safe and effective alternative treatment of some movement disorders such as Parkinson's disease. Although DBS is an effective treatment for Parkinson's disease, because of the necessity of surgical intervention, follow-up and the effects on symptoms, this study was carried out to determine the challenges, expectations and accomplishments of patients with DBS in Parkinson's disease. Materials and Methods. This qualitative study was carried out at the Neurosurgery Department of a research hospital in Turkey with seven patients who underwent DBS between 2008 and 2018. In the study, the challenges, expectations, and accomplishments of patients were investigated by using three focus group interviews in October 2018. Results. Among the participants, six patients were male, and one patient was female. The mean age of the patients was $56.85 \pm 16.48$. Three main themes were revealed in the study. These were (1) Reborn; decrease in dependence, sense of accomplishment, enjoyment of life, (2) Prejudice; perceived as severely ill by others and (3) Fear; not being accustomed to the device, loss of device function. Conclusion. The results obtained from this study can be used in the process of adaptation to this process by discussing and evaluating the challenges, expectations and accomplishments of the Parkinson's patient in DBS with healthcare professionals and other patients.
\end{abstract}

Key Words: Deep Brain Stimulation - Parkinson's Disease - Patient Expectation - Challenge - Accomplishments.

\section{Introduction}

The main and early symptoms of Parkinson's disease (PD) include bradykinesia/akinesia, rigidity, and rest tremor, while later symptoms are postural instability and walking and balance disorders, and there is a progressive movement disorder $(1,2)$. In addition to the general symptoms, there may be symptoms such as cognitive and behavioural disorders, communication problems, urinary problems, sexual dysfunction, falls, dependence in daily life activities, walking and balance problems, weight loss, excessive sweating, sleep disorders and impulse control disorders particularly pathological gambling (2-5).

Deep brain stimulation (DBS) was first performed in 1987 by Benabid et al. (6). It is a safe and effective alternative to treatment of some movement disorders (7) such as Parkinson's disease (8,9), tremor (10-12), dystonia (13) and some neurological and psychiatric diseases (14) such as epilepsy (15) and obsessivecompulsive disorder $(16,17)$. It is well-known that PD is caused by loss of nigrostriatal dopaminergic neurons due to disruption of the dopamine and acetylcholine balance in the basal ganglia $(18,19)$.

The procedure provides electrical stimulation by burning the brain regions such as the subthalamic nucleus, the globus pallidus internus and the ventralis intermediate nucleus of the thalamus, using high frequency sound waves. It reduces the activity of abnormally increasing cells, or by increasing dopamine activity through deep electrodes placed in this region, for preventing 
clinical signs and symptoms $(9,14,18,20)$. It does not cure the disease, but alleviates symptoms such as tremor, rigidity and bradykinesia, improves the quality of life of the patient, reduces or eliminates the amount of medication used, and provides independence in daily life activities $(5,14,21)$.

There are different opinions on DBS in the literature (22-25). Cabrera et al. (22) stated that psychosocial factors, genetics, safety and efficacy of different targets play a role in the patient's response to DBS. Kim et al. (23) reported that DBS is important in patients with high response to levodopa treatment in vascular Parkinsonism. Additionally, when they compared elderly and young patients who underwent DBS, they found more surgical complications in the elderly group, yet psychosis was seen more in the younger group, but this difference was not very significant. Therefore, the procedure was as applicable in the elderly as in younger individuals.

Mueller et al. (25) studied changes in brain connectivity while comparing the effects of DBS to those of levodopa treatment in $\mathrm{PD}$, and found that the treatment effect of DBS was associated with high connectivity inside the cerebello-thalamocortical network. They also found increased interconnectedness in the right and left motor cortices in patients treated by DBS. Little et al. (26), in their study on the effects of bilaterally adaptive DBS in Parkinson's patients, found a reduction in bradykinesia and axial symptoms. Mirzadeh et al. (27) determined that the mean off-medication motor scores and quality of life improved, and the levodopa equivalent daily dose was reduced in 6th months after DBS. They stated that there were no significant adverse events.

In the literature, it may be seen that studies have usually focused on clinical symptoms and procedures $(18,20,22-26)$. However, since each patient's emotions are different, their direct statements as first persons and explanations of their experiences can guide other patients and healthcare professionals. In addition, the number of patients is low in the hospital where the study is being conducted because it is not a common procedure for Parkinson's patients. So, in this study the views of Parkinson patients who had undergone DBS for ten years in the hospital were examined qualitatively.

The aim of this study was to determine the challenges, expectations, and accomplishments of patients with DBS in PD.

\section{Methods}

\section{Design and Participants}

This qualitative study was conducted with three focus group interviews including seven patients with Parkinson's disease treated with DBS. There were rest tremors in 7 patients, bradykinesia/ akinesia, urinary problems, sexual dysfunction, falls, dependence in daily life activities, and walking and balance problems in 5 patients, progressive movement disorder and weight loss in 2 patients, sleep disorders in 4 patients, and rigidity, cognitive and behavioural disorders and communication problems in 3 patients.

\section{Data Collection}

The data were collected in October 2018 through three focus group interviews using a semistructured interview to determine the experiences of the patients. This study received the necessary ethics approval from the Ethics Board of Zeynep Kamil Education and Research Hospital in Istanbul, Turkey (01.08.18/123). A total of ten patients who underwent DBS between 2008 and 2018 at the Neurosurgery Department of a research hospital in Turkey were reached by the researchers, and given information on the purpose of the study. The study was performed in accordance with the Declaration of Helsinki. Verbal consent was obtained from the patients for the focus group interviews.

A quiet and comfortable room was arranged at the hospital for the interview. Seven patients volunteered for the study, and three patients did not. Before the interview, tags were prepared for the participants, the moderator and the rapporteur. The moderator and the rapporteur came to the meeting area before the focus group interview 
and completed the preparations (table, chairs, papers, pencils, seating arrangement and food). The participants were also met by the moderator and the rapporteur. One recording device was prepared. Prior to the start of the interview, the moderator recorded the date, place, hour, who would manage the interview and the name of the rapporteur on the recording device. Thus, the device was checked. To prevent interruptions, a note was written on the outside of the door of the meeting room. While waiting for all the patients to arrive, nothing was discussed with the incoming participants. When the arrival of the patients was complete, the moderator stated that the interview would be recorded using a voice recorder. It was stated that these records would be examined by the researchers in the study, they would be used for scientific purposes only, and the names of the participants would not be included. The participants were asked to switch their mobile phones off before the interviews started. The patients were given a brief description of the purpose of the study and were provided information on the focus group interviews.

In the interviews, semi-structured questions were asked to clarify the experiences of the patients:

- What do you think about DBS? Would you recommend it to others?

- Did you experience any difficulties during and after DBS? What would you say?

- What do you think about the changes in your life after DBS?

Thus, the perspectives, experiences, thoughts, perceptions, feelings, attitudes and habits of the participants, and their psychological and social situations were evaluated in the three focus group interviews. The interviews lasted for 120 minutes.

\section{Data Analysis}

In this study, the content analysis method was used to analyse the data obtained from the interviews. In the content analysis, the data were coded first. The researchers analysed the data they obtained and divided them into meaningful categories. So, the conceptual expressions in these sections were examined. Then, each researcher created themes by coding the data. Finally, the researchers discussed the codes and themes together and agreed on the common themes.

\section{Results}

Among the participants, six patients were male, and one patient was female. The mean age of the patients was $56.85 \pm 16.48$ (30-78), and they had been diagnosed with Parkinson's disease $11.85 \pm 4.48$ (7-20) years ago. DBS was performed $6.14 \pm 2.34$ (3-9) years ago. The following themes emerged as a result of the interviews with the patients with Parkinson's disease: Reborn: Decrease in dependence; Sense of accomplishment; Enjoyment of life; Prejudice: Perceived as severely ill by others; Fear: Not being accustomed to the device; Loss of device function.

\section{Reborn}

In the study, the participants stated that DBS had given them a new life and identity, decreased their dependence on others, they had experienced a sense of recovery from the disease, and their enjoyment of life had increased.

Decrease in Dependence. The participants stated that DBS had significantly reduced their dependence on others, and they were able to continue their lives without need of others.

"...I was born again by this process. It is like I am in another body. My hands were shaky before. I had difficulties in walking...". Male, age: 42.

"...Now I ask for help from my loved ones less...". Male, age: 30 .

"...It is amazing how self-sufficient you are. First of all, thank God and then you (Doctor, nurse, etc.). I think the most important issue is its positive contribution to independence. Thank God...". Female, age: 64 .

"...The amount of medication I take decreased. It makes me happy, too...". Male, age: 54 .

Sense of Accomplishment. In this study, the participants thought that their disease would always get worse before DBS, and they were 
helpless regarding the disease. However, they stated that they now believed they would recover from PD, and they had the pleasure of being successful. Some expressions of the participants are included below:

"...I had been thinking that my life was going to get worse. But then I saw that this disease had a treatment. I am not scared anymore. Even if there are occasional problems, I can deal with these problems. I am confident...". Male, age: 60.

"...Both in this disease and other diseases, I do not feel helpless. They (other people) should read about it. They should also trust their doctors and nurses...". Male, age: 70.

"...A human being is such an entity that when you think you cannot get rid of this disease, the symptoms increase. You are worse. But when you see the survivors of this disease through this surgery, you can say "I can do it" with good reason...". Female, age: 64.

Enjoyment of Life. In this study, two participants stated that life before DBS was painful, and feelings of pessimism were dominant.

"...Parkinson's is a very bad disease. It always progresses. What is going on? You are worried that dependence will increase. This surgery gave me my smile back. I wish I had never had this disease. ...but I still feel good when I focus on my progress...". Male, age: 78 .

"...I had a really bad time. Of course, in such a serious disease, when I was not able to carry a glass of water, it was very bad when others finished what they were doing in a few seconds. Now I can carry my cup more easily. I can drink water by myself. How nice. When I drink my water easily from the cup, there is no happier person than me... . Male, age: 60 .

\section{Prejudice}

The participants stated that other people had prejudices about the surgery.

Perceived as Severely Ill by Others. In the study, three patients with PD stated that when the tool inserted into their brains, was felt by others, they perceived them as more severe patients than other people. Therefore, they reported that it would be better if this surgery was not visible from the outside. Some of what the participants said is given below:

"...Sometimes it seems to me that when people see me, they think I am sicker. It seems like it would be better if I did not let them know that I have had brain surgery.... Female, age: 64.

"...I wish it was not noticeable when somebody looks at me. It would be better...". Male, age: 54 .

"...When there is something in my head, even some of my relatives look at it strangely. ...but I also want them to care about me being well. It is more important for me to be well. Sometimes I think I can read their thoughts. Generally, when people have surgery, they reflect on the surgery as if they were more severe patients...". Male, age: 60 .

\section{Fear}

In the study, some Parkinson's patients stated that they had some fears. They stated that they were afraid of losing their good and nice side with DBS. Additionally, two participants stated that they were concerned that the battery would not function properly after the surgery.

Not Being Accustomed to the Device. In this study, the participants stated that they were afraid of perceiving the device as a foreign body and not getting used to the device.

"...Actually, my doctor told me about the process. ...but, you are carrying a device that does not belong to your body. I wondered if I would have problems...". Male, age: 42.

"...I tried to think I was going to be okay. So, I felt a bit of strangeness. Then I got used to it...". Male, age: 60 .

"...It is not just about this device. It is a foreign body inside you...". Female, age: 64.

Loss of Device Function. Four participants stated that they feared that the device would not work, and they were troubled by problems. The patients expressed fears about the potential failure of the battery. Some statements by the participants are included below:

"...My doctor explained it to me very well. I can contact him immediately if there are any 
problems. However, in some moments, I am afraid of the battery stopping suddenly, so, I would have problems again, which would mean I cannot even drink water, I worry...”. Male, age: 60 .

"...I had this fear more at first. There were always questions. I suppose I was scared of going back to my old self. Thank goodness, I rarely think about it now...". Male, age: 30.

\section{Discussion}

The results of our study focused on revealing the patients' challenges, expectations, and accomplishments rather than the effect of DBS on PD. In this study, the patients' feelings and opinions associated with their lives after DBS were included as their first-person experiences. As a result of this study, although the patients stated that they felt reborn, they said they had experienced prejudice and fear. We can say that DBS brings different challenges to patients, in addition to good outcomes. Gorman and Sultan (28) reported that evaluation is important not only of physical outcomes but also patients' experiences, difficulties and expectations.

According to the results of our study, the patients were satisfied with the DBS procedure, and they stated that they felt born again. As a result of this study, after DBS in Parkinson's patients, there were improvements in walking, they asked for help from others less, and they were more independent. Diseases such as PD, which cause physical movement disorders and psychological negativity, necessitate long-term treatment and the use of drugs in individuals. Additionally, the patient's dependence on others increases over the years despite treatment. This causes psychological problems, such as tiredness, exhaustion, depression and anxiety in patients and their relatives. These negative effects on the patient and their caregivers cause a decrease in the patient's progress in treatment, and lower their expectations and motivation in their struggle against difficulties $(29,30)$. Healthcare includes a holistic assessment of the patient and anticipating the problems that may or may not be present in the patient. Therefore, evaluation of the physical and psychological status of Parkinson's patients and their dependency on others after DBS may improve the patient's adaptation capacity and develop their ability to cope with difficulties. Faivre et al. (29), in their animal model, stated that psychological disorders, such as anxiety and depression are seen in Parkinson's patients. Suzuki et al. (30) reported that the psychological problems of individuals with chronic diseases increased.

In the study, some participants stated that DBS stimulated their sense of accomplishment and their belief that they could cope with problems. The patients treated with DBS indicated that they felt happier because of its positive contribution to their well-being. Avanzino et al. (31) reported that the emotions of Parkinson's patients are important, and a lack of depression and anxiety in patients may increase walking speed, arm-swing, flexion of posture and step length, and provide initiation reaction time on patient.

Another result of this study is that patients stated that before DBS, life was painful, and they felt pessimistic. However, they stated that they were now very happy when they were able to perform their daily life activities independently and were less reliant on others. These results are supported by the literature. Lin et al. (32), in their study on 6-year follow up of patients' expectations and satisfaction with DBS for Parkinson's disease, determined that the quality of life of DBS patients increased due to the increase in mobility and daily activities, and additionally, all patients in the study felt they had made the right decision about DBS, and they would recommend DBS to others. The researchers also stated that the satisfaction levels of patients treated with DBS were always found to be high. Buhmann et al. (33) reported that patients treated with DBS were more successful in driving stimulation than those treated without DBS, and their cognitive abilities were better after DBS. Unlike the results of this study, Maier et al. (34) reported no improvement in the quality of life of patients after DBS.

In this study, some patients stated that DBS was perceived as serious surgery by others because it 
was a surgical procedure on the brain. They thought it created prejudice against them. In particular, interventions made in the brain and central nervous system are more important because they are complex and directly affect the individual's vital functions. These procedures are important for the survival of patients. Therefore, surgical interventions on the brain are classified by the community as severe. Negative attitudes and prejudice against Parkinson's patients cause psychiatric problems. Therefore, these patients should benefit more from social support systems (35). Contrary to the belief expressed in this study that patients are perceived as severely ill by other individuals because of the surgical procedure, Vergani et al. (24), in their study evaluating 141 Parkinson's patients, stated that DBS was a safe procedure, had low mortality rates, and surgical complications were rare.

In the study, some Parkinson's patients stated that they were afraid that they would not get used to the DBS device and worried about the potential of the battery losing its function. Although they stated that they had received information and support from DBS-related healthcare professionals, they mentioned that these fears arose especially in the early stages. Although the applicability of the programmable pulse generator which was implanted in DBS was approved (22), patients' fear of the device and the battery losing its function may be associated with individual factors and healthcare. Unlike our study, Kim et al. (36) reported that patients with PD who underwent DBS had fears of complications and the economic burden. In order to understand the causes of fear, Gorman and Sultan recommended that the patients' personal reactions, maximum powers, factors affecting objectivity and psychological status should be examined (28).

\section{Limitations of the Study}

The limitation of this study was that the study was conducted in a single centre with seven patients. The results are therefore valid only for the population of the study. In order to generalise the results, different centres and groups should be included.

\section{Conclusion}

According to the results of this study, DBS contributes positively to the daily living activities of patients with $\mathrm{PD}$, and increases their sense of reattachment and hope. However, as in any surgical procedure, patients experience fear. Therefore, in future studies, it may be recommended to establish protocols for adequate counselling for patients before the DBS surgery. The results of this study may be used for this kind of protocol. There was no specific questionnaire available to assess the quality of life of people with PD who underwent DBS. This focus group interview results could be used to structure a new questionnaire to assess the quality of life of people with PD who have undergone DBS. Also, support groups for patients with PD may help them to cope mentally with the burdens of the disease.

\section{What Is Already Known on this Topic:}

Parkinson's disease (PD) is a common chronic neurodegenerative disease. In PD, motor symptoms are well known, and treatments (pharmacological / physiotherapy / surgical) are mostly focused on this area. As a surgical treatment, deep brain stimulation (DBS) is a safe and effective alternative to treatment of some movement disorders such as PD. $D B S$ does not cure the disease, but alleviates the symptoms, improves the quality of life of the patient, reduces or terminates the amount of drugs used, and provides independence in daily living activities. In the literature, studies have usually focused on clinical symptoms and procedures.

\section{What this Study Adds:}

The success of treatment with DBS cannot be seen only in the initial recovery after surgery. Most patients require bio-psycho-social adaptation to their new experiences in life with implanted electronic devices. They should also be informed about their fears, and the possibilities and limitations of the treatment. As each patient's emotions are different, healthcare professionals must provide support to patients and caregivers in the pre- and post-operative period, by sharing their knowledge and experience, addressing the challenges, expectations, and accomplishments of the patients in a holistic manner, as stated in the results of this study.

Authors' Contributions: Conception and design: Öİ, SM and EA; Acquisition, analysis and interpretation of data: Öİ, SM and EA; Drafting the article: Öİ and SM; Revising it critically for important intellectual content: Öİ and SM; Approved final version of the manuscript: Öİ, SM and EA.

Conflict of Interest: The authors declare that they have no conflict of interest. 


\section{References}

1. Parkinson J. An essay on the shaking palsy. 1817. J Neuropsychiatry Clin Neurosci. 2002;14(2):223-36; discussion 222.

2. Jankovic J. Parkinson's disease: clinical features and diagnosis. J Neurol Neurosurg Psychiatry. 2008;79(4):368-76.

3. Combs HL, Garcia-Willingham NE, Berry DTR, van Horne CG, Segerstrom SC. Psychological functioning in Parkinson's disease post-deep brain stimulation: Selfregulation and executive functioning. J Psychosom Res. 2018;111:42-9.

4. Pfeiffer RF. Non-motor symptoms in Parkinson's disease. Parkinsonism Relat Disord. 2016;22(1):119-22.

5. Altuğ F, Acar F, Acar G, Cavlak U. The influence of subthalamic nucleus deep brain stimulation on physical, emotional, cognitive functions and daily living activities in patients with Parkinson's Disease. Turk Neurosurg. 2011;21(2):140-6.

6. Benabid AL, Pollak P, Louveau A, Henri S, de Rougemont J. Combined (thalamotomy and stimulation) stereotactic surgery of the VIM thalamic nucleus for bilateral Parkinson disease. Appl Neurophysiol. 1987;50(1-6):344-6.

7. Siddiqui JH, Bhatti D, Alsubaie F, Bajwa JA. Movement Disorders and Deep Brain Stimulation in the Middle East. World Neurosurg. 2018;113:e314-9.

8. Timmermann L, Jain R, Chen L, Maarouf M, Barbe MT, Allert N, et al. Multiple-source current steering in subthalamic nucleus deep brain stimulation for Parkinson's disease (the VANTAGE study): a non-randomised, prospective, multicentre, open-label study. Lancet Neurol. 2015;14(7):693-701.

9. Rizzone MG, Martone T, Balestrino R, Lopiano L. Genetic background and outcome of Deep Brain Stimulation in Parkinson's disease. Parkinsonism Relat Disord. 2019;64:8-19.

10. Oliveria SF, Rodriguez RL, Bowers D, Kantor D, Hilliard JD, Monari EH, et al. Safety and efficacy of dual-lead thalamic deep brain stimulation for patients with treatment-refractory multiple sclerosis tremor: a single-centre, randomised, single-blind, pilot trial. Lancet Neurol. 2017;16(9):691-700.

11. Sandoe C, Krishna V, Basha D, Sammartino F, Tatsch J, Picillo M, et al. Predictors of deep brain stimulation outcome in tremor patients. Brain Stimul. 2018;11(3):592-9.

12. Rebelo P, Green AL, Aziz TZ, Kent A, Schafer D, Venkatesan L, et al. Thalamic Directional Deep Brain Stimulation for tremor: Spend less, get more. Brain Stimul. 2018;11(3):600-6.

13. Volkmann J, Mueller J, Deuschl G, Kühn AA, Krauss JK, Poewe W, et al. Pallidal neurostimulation in patients with medication-refractory cervical dystonia: a randomised, sham-controlled trial. Lancet Neurol. 2014;13(9):875-84.
14. Halpern C, Hurtig H, Jaggi J, Grossman M, Won $\mathrm{M}$, Baltuch G. Deep brain stimulation in neurologic disorders. Parkinsonism Relat Disord. 2007;13(1):1-16.

15. Salanova V. Deep brain stimulation for epilepsy. Epilepsy Behav. 2018;88S:21-4.

16. Kohl S, Baldermann JC. Progress and challenges in deep brain stimulation for obsessive-compulsive disorder. Pharmacol Ther. 2018;186:168-75.

17. Welter ML, Houeto JL, Thobois S, Bataille B, Guenot M, Worbe Y, et al. Anterior pallidal deep brain stimulation for Tourette's syndrome: a randomised, double-blind, controlled trial. Lancet Neurol. 2017;16(8):610-9.

18. Luoma J, Pekkonen E, Airaksinen K, Helle L, Nurminen J, Taulu S, et al. Spontaneous sensorimotor cortical activity is suppressed by deep brain stimulation in patients with advanced Parkinson's disease. Neurosci Lett. 2018;683:4853.

19. Ulusoy EK, Ayar E, Bayındırlı D. Facial emotion recognition and discrimination deficit in idiopatic Parkinson patients. Turkish Journal of Neurology. 2015;21(1):16-21.

20. Brahimaj B, Kochanski RB, Sani S. Microelectrode accuracy in deep brain stimulation surgery. J Clin Neurosci. 2018;50:58-61.

21. Follett KA, Weaver FM, Stern M, Hur K, Harris CL, Luo P, et al. Pallidal versus subthalamic deep-brain stimulation for Parkinson's disease. N Engl J Med. 2010;362(22):207791.

22. Cabrera LY, Sarva H, Sidiropoulos C. Perspectives on the Earlier Use of Deep Brain Stimulation for Parkinson Disease from a Qualitative Study of U.S. Clinicians. World Neurosurg. 2019;128:e16-20.

23. Kim M, Cho KR, Park JH, Ahn JH, Cho JW, Park S, et al. Bilateral subthalamic deep brain stimulation is an effective and safe treatment option for the older patients with Parkinson's disease. Clin Neurol Neurosurg. 2018;173:182-6.

24. Vergani F, Landi A, Pirillo D, Cilia R, Antonini A, Sganzerla EP. Surgical, medical, and hardware adverse events in a series of 141 patients undergoing subthalamic deep brain stimulation for Parkinson disease. World Neurosurg. 2010;73(4):338-44.

25. Mueller K, Jech R, Růžička F, Holiga Š, Ballarini T, Bezdicek $\mathrm{O}$, et al. Brain connectivity changes when comparing effects of subthalamic deep brain stimulation with levodopa treatment in Parkinson's disease. Neuroimage Clin. 2018;19:1025-35.

26. Little S, Beudel M, Zrinzo L, Foltynie T, Limousin P, Hariz $M$, et al. Bilateral adaptive deep brain stimulation is effective in Parkinson's disease. J Neurol Neurosurg Psychiatry. 2016;87(7):717-21.

27. Mirzadeh Z, Chapple K, Lambert M, Evidente VG, Mahant P, Ospina MC, et al. Parkinson's disease outcomes after intraoperative CT-guided "asleep" deep brain stimulation in the globus pallidus internus. J Neurosurg. 2016;124(4):902-7. 
28. Gorman LM, Sultan DF. Psychosocial Nursing for General Patient Care. 3rd edition. Philadelphia: F.A. Davis Company; 2008.

29. Faivre F, Joshi A, Bezard E, Barrot M. The hidden side of Parkinson's disease: Studying pain, anxiety and depression in animal models. Neurosci Biobehav Rev. 2019;96:33552.

30. Suzuki R, Nakamiya Y, Watanabe M, Ando E, Tanichi M, Koga M, et al. Relationship between stress coping mechanisms and depression in kidney transplant recipients. Transplant Proc. 2019;51(3):761-7.

31. Avanzino L, Lagravinese G, Abbruzzese G, Pelosin E. Relationships between gait and emotion in Parkinson's disease: A narrative review. Gait Posture. 2018;65:57-64.

32. Lin HY, Hasegawa H, Mundil N, Samuel M, Ashkan K. Patients' expectations and satisfaction in subthalamic nucleus deep brain stimulation for Parkinson disease: 6-year follow-up. World Neurosurg. 2019;121:e654-60.
33. Buhmann C, Maintz L, Hierling J, Vettorazzi E, Moll CK, Engel AK, et al. Effect of subthalamic nucleus deep brain stimulation on driving in Parkinson disease. Neurology. 2014;82(1):32-40.

34. Maier F, Lewis CJ, Horstkoetter N, Eggers C, Kalbe E, Maarouf M, et al. Patients' expectations of deep brain stimulation, and subjective perceived outcome related to clinical measures in Parkinson's disease: a mixedmethod approach. J Neurol Neurosurg Psychiatry. 2013;84(11):1273-81.

35. Lee SJ, Kim SR, Chung SJ, Kang HC, Kim MS, Cho SJ, et al. Predictive model for health-related quality of life in patients with Parkinson's disease. Geriatr Nurs. 2018;39(2):204-11.

36. Kim MR, Yun JY, Jeon B, Lim YH, Kim KR, Yang HJ, et al. Patients' reluctance to undergo deep brain stimulation for Parkinson's disease. Parkinsonism Relat Disord. 2016;23:91-4. 